Regards sur l'économie allemande

Bulletin économique du CIRAC

$69 \mid 2004$

Varia

\title{
IG Metall : une nouvelle politique dans le champ social
}

\section{CpenEdition}

\section{Journals}

Édition électronique

URL : https://journals.openedition.org/rea/3443

DOI : $10.4000 /$ rea.3443

ISBN : 978-2-8218-0834-8

ISSN : 1965-0787

Éditeur

CIRAC

Édition imprimée

Date de publication : 1 décembre 2004

ISSN : 1156-8992

Référence électronique

"IG Metall : une nouvelle politique dans le champ social », Regards sur l'économie allemande [En ligne], 69 | décembre 2004, mis en ligne le 27 janvier 2009, consulté le 28 juin 2022. URL : http:// journals.openedition.org/rea/3443; DOI : https://doi.org/10.4000/rea.3443

Ce document a été généré automatiquement le 29 septembre 2020

(C) CIRAC 


\section{IG Metall : une nouvelle politique dans le champ social}

1 Le syndicat IG Metall cherche à se repositionner en tant qu'acteur politique en investissant le champ de la protection sociale et de ses réformes. En prévision de son «Congrès sur l'Etat social » programmé les 6 et 7 avril 2005 à Berlin, le syndicat élabore ses propres propositions de réformes en matière de financement des systèmes de retraites et d'assurance maladie, sans négliger pour autant le cœur de sa mission : la régulation du marché de l'emploi. Ces propositions, en cours de rédaction, seront soumises à débat sous forme de memorandum. "L'Etat social doit évoluer. Le statu quo n'est plus tenable: il ne permet ni de répondre aux défis actuels, ni encore moins d'assurer la pérennité du système », nous explique dans un entretien Wolfgang Schröder, responsable du département Politique sociale d'IG Metall renforcé l'an dernier dans le cadre du processus de refondation (voir REA 58/02).

\section{Santé : plus de concurrence entre caisses publiques et privées}

2 Dans le domaine de l'assurance maladie, «il ne suffit plus de réformer le système à la marge. Il y a un fort consensus en Allemagne sur ce point : il faut un changement de paradigme ». Pour " mettre fin à la médecine de classe », IG Metall plaide ainsi lui aussi pour une "assurance citoyenne » (Bürgerversicherung), c'est-à-dire un mode de prélèvement frappant tous les actifs et tous les revenus, sans distinction (voir REA $63 / 03$ et 68/04). Ces cotisations, qui ne seront donc plus assises sur le seul travail salarié, seront versées obligatoirement au système public d'assurance maladie, y compris par les fonctionnaires et les travailleurs indépendants, c'est-à-dire ceux dont les revenus salariés sont supérieurs au plafond de l'assurance légale et qui s'assurent donc auprès des caisses privées. Cela devrait permettre d'accroître l'assiette du financement des caisses légales et de réduire leur déficit. De la sorte, «les caisses publiques verront s'améliorer leur compétitivité face aux caisses privées ». Car il s'agit de maintenir le principe du libre choix de l'affiliation et de constituer à terme deux pôles de force équivalente " pour enfin permettre une concurrence digne de ce nom ». 
Ce sont là ni plus, ni moins, les thèses que défend le Conseil des Sages depuis son rapport de 2002 sur la situation de l'économie allemande (voir REA 59/02). On voit là que, sous le discours syndical convenu, pointe un solide pragmatisme.

\section{Hartz IV : critique sur l'abandon du concept de métier}

3 Dans le domaine de la régulation du travail, les positions du directoire d'IG Metall sont plus nuancées. D'un côté, le syndicat salue expressément les réformes Hartz, «les plus fondamentales depuis plus de trente ans». Mais s'il approuve la suppression de l'un des trois étages de l'ancien système d'indemnisation du chômage (l'Arbeitslosenhilfe, voir REA 68/04), puisque "cela réduit la bureaucratie et permet aux bénéficiaires de l'aide sociale de participer à des mesures de réinsertion en emploi », il désapprouve le fait que le montant du nouveau revenu minimum d'insertion (Arbeitslosengeld II) est identique à celui de l'aide sociale. Ce n'est pas tant le montant de l'aide de base ( $345 €$ à l'ouest) qu'incrimine le syndicat; il s'insurge contre l'obligation faite au chômeur d'accepter tout emploi proposé par son conseiller, même si la rémunération est inférieure aux normes en vigueur dans la branche et même si l'emploi ne correspond pas aux qualifications initiales du candidat. Le second grief pèse beaucoup plus lourd que le premier, car il vide de sa substance l'un des principes fondateurs non seulement du syndicalisme allemand, mais plus généralement encore de l'organisation du travail : ce concept de métier qui assurait jusqu'ici l'intégration professionnelle et sociale via les qualifications professionnelles (voir dans ce numéro). Il s'agira donc de mener une offensive sur les qualifications.

\section{Pour un segment à bas salaires}

4 Mais si son syndicat a malgré tout donné son aval aux lois Hartz, c'est au nom d'un autre principe (ou valeur), supérieur: "le travail est la clef de l'intégration sociale». L'individu doit pouvoir réaliser cette intégration par ses propres forces et moyens. Or ce préalable n'était plus donné : les mesures de traitement social du chômage se sont révélées impuissantes à endiguer un chômage structurel de longue durée. "Cette situation n'était plus défendable", estime-t-on à IG Metall. Elle l'était d'autant moins qu'elle aboutissait à un effet pervers, interdisant aux moins qualifiés de s'intégrer dans la vie professionnelle. Or les mesures Hartz s'adressent aussi à ceux-là, en " ouvrant un segment à bas salaires pour les personnes en mal d'employabilité " grâce aux mesures de requalification. Voilà comment, au nom même des principes fondateurs de l'économie sociale de marché, le syndicat IG Metall en est amené à rompre avec certains de ses tabous.

\section{Flexibilisation des conditions de travail, mais...}

5 En matière de conditions du travail, l'approche est similaire. Après tout, estime-t-on, il faut être réaliste face à l'érosion du travail normal, et accepter dans le même temps que l'idéal du plein emploi relève aujourd'hui du mythe. Dès lors, l'allongement de la durée du travail ne constitue pas une rupture avec le dogme - à condition qu'il s'agisse d'accords isolés, liées à des situations spécifiques (Siemens...). «L'évolution est un défi que 
nous nous devons de relever", explique W. Schroeder en ajoutant toutefois "qu'il est hors de question de généraliser ce type d'accords au niveau de la branche ou de l'industrie». IG Metall n'est pas le seul à refuser une hausse uniforme et généralisée du temps de travail, le patronat, les fédérations professionnelles et la plupart des économistes lui préfèrent eux aussi la flexibilisation des conditions de travail. Elle n'en constitue pas moins un risque pour la structure de la régulation sociale allemande. Il se manifeste doublement dans la multiplication d'accords catégoriels (que prône, pas toujours de son propre gré, le syndicat ver.di) et d'accords d'entreprise (pratiquée activement par IG Metall ces derniers mois). Il s'agit bien là d'évolutions aux conséquences irréversibles, impliquant une refondation de longue haleine du partenariat social allemand...

\section{Retraites : pour plus de capitalisation}

Plus urgente est la question des retraites. Sans réforme, "nous serons bientôt confrontés à la paupérisation des personnes âgées, un grave problème de société». L'introduction d'éléments de capitalisation depuis la réforme Riester (2000), " nous l'acceptons, explique W. Schroeder, mais à conditions que la garantie du standard de vie repose sur les trois piliers" que sont le régime des cotisations, un complément de capitalisation, et un système de retraites d'entreprises. La réforme devant "bien entendu être compatible avec les impératifs macro-économiques", IG Metall refuse d'envisager une fiscalisation plus poussée du financement des caisses de retraites : « $30 \%$, comme actuellement, c'est déjà trop!».

\section{MetallRente, un fonds de pension de branche}

7 La solution pourrait s'inspirer du modèle suédois, où un complément de capitalisation est obligatoire. Car le problème actuel de la retraite Riester est sa faible diffusion: guère plus de 4 millions de contrats ont été conclus à ce jour; or ils sont destinés à compenser la baisse programmée des pensions de retraite versées... Visiblement, les Allemands redoutent la complexité de ce produit financier; ils surestiment aussi leurs revenus de retraités, et ils ont un problème culturel lié à l'argent (voir REA 67/04). La problématique des retraites sera donc le thème-clef du congrès d'avril prochain. $\mathrm{Ce}$ sera aussi l'occasion de faire de la publicité pour MetallRente, le fonds de pension mutualiste propre à la métallurgie (et surtout au Mittelstand), créé conjointement en 2001 par le syndicat et son partenaire patronal Gesamtmetall. La création était une réponse au refus des entreprises de la branche de constituer des fonds de retraite d'entreprises. Or MetallRente ne compte encore que 120000 souscripteurs, de surcroît dans les tranches de revenus supérieures.

\section{Durée de la vie professionnelle : 45 ans au maximum !}

Il faut aussi élargir l'assiette. Mais «il est moins urgent en ce moment de hausser l'âge légal de départ à la retraite que l'âge moyen effectif». Le syndicat plaide pour une grande flexibilité (un corridor allant de 62 à 65 ans) et une gestion au cas par cas, et selon la pénibilité du travail. Mais " 45 ans de vie professionnelle, c'est pour nous un maximum 
absolu!». Une limite somme toute réaliste, sachant que, si la durée moyenne de la vie professionnelle des hommes est de 40 ans à l'ouest, toutes branches confondues, presque la moitié des salariés ( $40 \%$ ) travaille 45 ans ou plus (mais seulement 3,6\% des femmes à l'ouest). S'il faut reconsidérer la place des salariés âgés dans l'entreprise en valorisant notamment leur expérience et leur créativité, il faut surtout élargir l'assiette des cotisations, en l'étendant à tous les actifs, occupés ou non.

\section{Pour la modernisation du modèle bismarckien}

9 "Dans l'intérêt de nos membres comme dans celui de notre crédibilité, nous devons formuler une position autonome " dans le débat public lié à l'Agenda 2010, expliquait son premier président, Jürgen Peters, lors d'un congrès d'IG Metall en octobre dernier. Cogestionnaire depuis toujours des caisses de retraite et d'assurance maladie (comme tous les syndicats), IG-Metall veut dorénavant co-définir les options de modernisation du modèle bismarckien. C'est un peu comme si cet acteur établi de la macro-régulation sociale allemande redécouvrait la mission d'interlocuteur institutionnel qui revient pourtant traditionnellement outre-Rhin au syndicalisme en général, et au DGB en particulier, dans le jeu collectif de la définition de choix sociétaux.

\section{Pour une alternative politique}

Il est vrai que diverses évolutions l'y incitent vivement. D'abord, le défi que posent au syndicalisme allemand et au jeu du partenariat social les mutations des activités qui brouillent les délimitations de branche et mettent à mal le fondement de la macrorégulation sociale qu'est la convention de branche; pour IG Metall, la tertiarisation des activités se solde par la réduction du nombre de ses adhérents. Ensuite, la concurrence du géant des services ver.di qui, s'il est toujours en quête de culture propre (voir REA $59 / 02$ ) après sa fusion, mène une stratégie politique d'actions ponctuelles, mettant à profit le fonds idéologique altermondialiste naissant outre-Rhin. Ensuite, de l'aveu même de Jürgen Peters, son organisation n'a pas réussi, au cours des deux années écoulées, à «ancrer dans la tête et le cœur » des Allemands une alternative politique. Les raisons officiellement avancées: une "relation mal définie» avec le gouvernement de coalition rouge-vert, et « une coordination insuffisante » entre "l'action dans l'entreprise et le positionnement politique».

\section{Regagner la confiance de la base}

11 En réalité, la base d'IG Metall, de culture encore largement 'métallo', ne reconnaît plus 'son' SPD (et hue le chancelier) qui mène à son sens une politique 'libérale'. Un conflit couve ainsi entre une base qui subit sur le terrain les effets de la stagnation comme des réformes sociales et une direction que son analyse macro-économique des mutations amène à partager globalement les grandes orientations des réformes du gouvernement fédéral. C'est aussi pour retrouver la confiance de sa base que la direction d'IG Metall prend l'initiative politique pour réformer un système de protection sociale qui, ainsi que le résume W. Schroeder, s'il se montre "généreux au plan des transferts, n'œuvre guère pour la justice sociale ». (IB) 